\title{
Fluid Dynamics in Normal and Stenosed Human Renal Arteries: an Experimental and Computational Study *
}

\author{
Fuyou LIANG $^{* *}$ Ryuhei YAMAGUCHI ${ }^{* * *}$ and Hao LIU ${ }^{* *}$ \\ ${ }^{* *}$ Graduate School of Science and Technology, Chiba University, \\ 1-33 Yayoi-cho, Inage-ku, Chiba, Japan \\ E-mail: hliu@faculty.chiba-u.jp \\ ***Department of Mechanical Engineering, Shibaura Institute of Technology, \\ 3-9-14 Shibaura, Minato-ku, Tokyo, Japan
}

\begin{abstract}
Atherosclerotic renal artery stenosis (RAS), which accounts for approximately 7\% of peripheral vascular diseases, is considered a major cause for secondary hypertension and other renal complications such as chronic renal failure and ischemic nephropathy. In this study, the fluid dynamic features of a human aortorenal bifurcation are investigated in detail with a computational fluid dynamics (CFD) solver to assess the localization of RAS in relation to the sites exposed to abnormal hemodynamic events. Specially, a normal renal artery is artificially rendered stenosed to examine the RAS-induced hemodynamic changes and their effect on the progression of RAS. The CFD solver is partially validated by a model experiment conducted for steady state flow. The computed results indicate that low oscillatory wall shear stress (WSS), which stands for the most prominent hemodynamic factor responsible for atherosclerosis, correlates intimately with flow separation; WSS distribution depends significantly on vascular geometric structure; and RAS may elicit pronounced flow disturbances that are likely to promote the spread of atherosclerotic lesions towards downstream region.
\end{abstract}

Key words: Fluid Dynamics, Renal Artery Stenosis, CFD, Wall Shear Stress, Mean Wall Shear Stress, Oscillatory Stress Index

\section{Introduction}

RAS is the most common cause for secondary hypertension and may lead to chronic renal failure and renal tissue injury ${ }^{(1)}$. The prevalence of RAS, such as the one shown in figure 1, occurs primarily in the upstream portion 1 to $2 \mathrm{~cm}$ distal to the renal ostium ${ }^{(3)}$. Most of renal artery lesions are associated with atherosclerosis. Etiological factors related to this degenerative vascular disease are complex and involve multiple mechanisms ranging from the individual-specific genetic make-up, diet and lifestyle to the local arterial hemodynamics ${ }^{(4)}$. Regardless of the very many factors, the observation that atherosclerotic plaques, stenoses or aneurysms typically present at some specific vascular locations like arterial bifurcations and bends has nowadays led to an almost universal acceptance of the hypothesis that local hemodynamics play a significant role in the onset, localization, and, perhaps more importantly, the progression of vascular disease in the human arterial system $^{(5)}$. Driven by this hypothesis, in the last decades, much effort has been undertaken in an attempt to identify hemodynamic factors responsible for the initiation and progression of vascular disease. However, to date much yet remains unclear regarding the relative importance and relevance of the many hemodynamic factors ${ }^{(5)}$. Despite this fact, many studies have reached a prevalent conclusion that WSS and its derivations, which act directly on the endothelium, play dominant roles in the onset and distribution of atherosclerotic 
lesions ${ }^{(4)}$

A large amount of studies concerning different aspects of renoarterial hemodynamics have been carried out. These studies include not only experimental studies performed either in vivo or in vitro or in simple idealized models, such as the model experiments ${ }^{(6)}$, and the in vivo measurements in human ${ }^{(3)}$, but also simulation studies such as an early simple model study ${ }^{(7)}$ and the recent medical-image-based CFD simulations ${ }^{(8,9)}$. So far, most of the experimental studies were confined

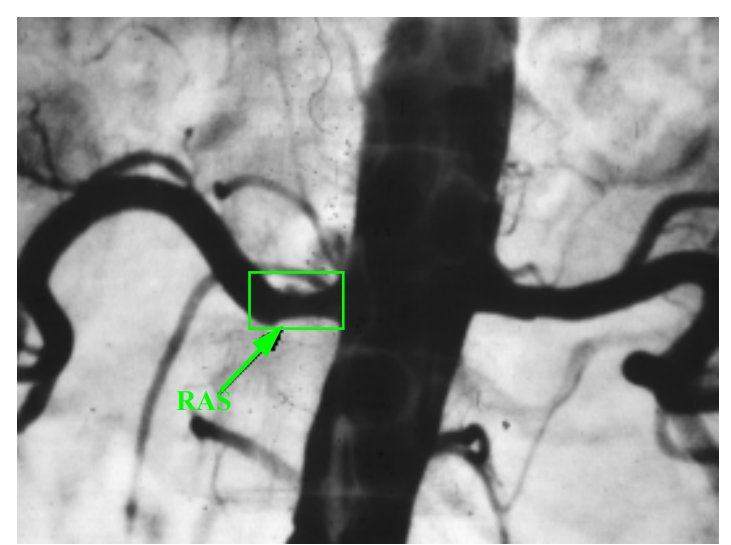

Fig.1 Renal artery angiogram after angioplasty surgery for $\operatorname{RAS}^{(2)}$ in steady state flow or ideal transient flow tests in simplified models. Although in vivo measurements can offer direct and realistic insights into in vivo phenomena; in practice, they are usually time-consuming, lacking in high resolution, and in most of the cases, limited to two-dimensional information. Contrastively, CFD-based computations may visualize and quantify hemodynamic variables of interest in a more straightforward and more detailed way. Till now, however, most of the computation studies focused exclusively on normal renal arteries free from vascular disease ${ }^{(8,9)}$. In fact, for a diseased renal artery suffering from RAS, how RAS alters the local hemodynamics and in turn how the altered hemodynamics affects the development of the existing RAS has long been a problem of special interest for clinical practice. To our knowledge, this topic yet remains rarely appreciated and less discussed in studies relevant to intravascular fluid dynamics.

The goal of the present study is twofold. The first is to quantify the renoarterial fluid dynamics to assess hemodynamic factors associated with RAS. The second is to evaluate the mutual effect between the progression of RAS and the RAS-induced changes in local hemodynamic condition. To this end, a finite volume method technique-based in house CFD solver was used. For solver validation, a model experiment was devised and conducted for steady state flow, where velocities were measured via laser Doppler anemometry (LDA) and wall shear stresses were measured with an electrochemical method.

\section{Materials and Methods}

\subsection{Model experiment}

\subsubsection{Aortorenal bifurcation model}

The configuration of the experimental aortorenal bifurcation model is illustrated in figure 2 , where the left and right renal arteries are identified by the angle from which the picture is viewed. The geometric parameters of the abdominal aortic trunk and the left renal artery were derived from a set of medical images ${ }^{(6)}$, while the right renal artery was designed to be an isodiametric straight tube. The branch/trunk area ratios of the two branches are the same at the outlets. In figure 2, the coordinate system and the WSS measuring positions are shown as well. The location where the $x$ axis intersects with the $y$ axis was defined as the origin point, and the plane formed by the two axes was named the common median plane which was oriented horizontally in the experiment.

\subsubsection{Measuring methods}

\section{(1) Velocity measurement}

Velocity measurement at the bifurcation was performed by using LDA. The convex lens of LDA had a focal length of $122 \mathrm{~mm}$, and the sampling volume formed by the intersection of two beams was in a shape of prolate spheroid with a diameter of about $0.04 \mathrm{~mm}$ and a 
length of about $0.3 \mathrm{~mm}$. The entering angle of laser beams to the common median plane was set at $90^{\circ}$. The measurements were done at intervals of $1 \mathrm{~mm}$ across the tube diameter, which results in a spatial resolution of the LDA measurement equal to the laser beams at the focal point.

The working fluid used for velocity measurement was the saturated aqueous potassium thiocyanate KSCN solution. The reason this fluid was selected was

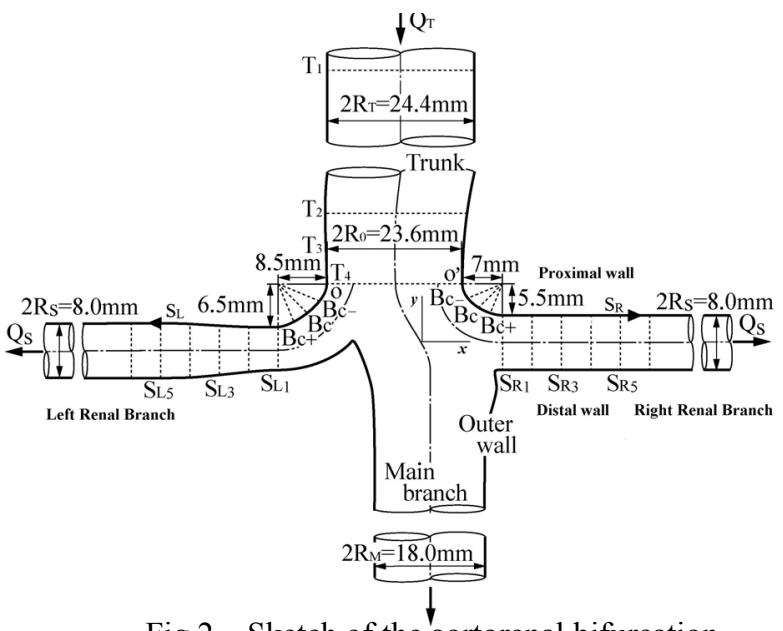

Fig.2 Sketch of the aortorenal bifurcation based on the consideration that its refractive index is equal to that of the acrylic plate used to produce the bifurcation model. The basic physical features of the fluid at a temperature of $298 \mathrm{~K}$ are listed below: the refractive index, $n=1.49$, the density, $\rho=1.44 \times 10^{3} \mathrm{~kg} / \mathrm{m}^{3}$, and the kinematic viscosity, $v=2.18 \times 10^{-6} \mathrm{~m}^{2} / \mathrm{s}$.

(2) WSS measurement

Wall shear stresses were measured via the 100 platinum electrodes embedded in the tube walls by acrylic cement. The mechanism underlying the measurement is that WSS is proportional to the cube of the electric current flowing between a test electrode and a counter electrode. To make the electrochemical method-based WSS measurement feasible, an electrolytic solution was used as the working fluid, which was composed of $0.01 \mathrm{M}$ potassium ferricyanide and $1.0 \mathrm{M}$ potassium hydroxide.

\subsection{Computational fluid dynamics and computer model construction}

\subsubsection{Governing equations and numerical algorithm}

The flow through the aortorenal bifurcation was assumed homogeneous, incompressible and Newtonian, the governing equations of which were the unsteady Navier-Stokes equations written in a strong conservation form for mass and momentum. The artificial compressibility method was utilized by adding a pseudo-time derivation of pressure to the continuity equation so that the elliptic nature of the equations can be relaxed to yield a hyperbolic-parabolic system. For an arbitrary computational cell $V(\mathrm{t})$, the governing equations are written as:

$$
\int_{V(t)} \frac{\partial \mathbf{q}}{\partial \tau} d V+\frac{\partial}{\partial t} \int_{V(t)} \mathbf{Q} d V+\oint_{S(t)} \mathbf{f} \cdot \mathbf{n} d S=0
$$

where $t$ and $\tau$ are the physical time and the pseudo-time respectively; $\mathbf{n}$ is the unit outward normal vector corresponding to the faces of the polyhedron cell; and the matrix vector is expressed as $\mathbf{f}=\left(\mathbf{F}+\mathbf{F}_{v}, \mathbf{G}+\mathbf{G}_{v}, \mathbf{H}+\mathbf{H}_{v}\right)$.

$$
\begin{aligned}
& \mathbf{Q}=\left[\begin{array}{l}
u \\
v \\
w \\
0
\end{array}\right] \quad \mathbf{q}=\left[\begin{array}{l}
u \\
v \\
w \\
p
\end{array}\right] \quad \mathbf{F}=\left[\begin{array}{c}
u^{2}+p \\
u v \\
u w \\
\beta u
\end{array}\right] \quad \mathbf{G}=\left[\begin{array}{c}
v u \\
v^{2}+p \\
v w \\
\beta v
\end{array}\right] \quad \mathbf{H}=\left[\begin{array}{c}
w u \\
w v \\
w^{2}+p \\
\beta w
\end{array}\right] \\
& \mathbf{F}_{v}=-\left(\frac{1}{\operatorname{Re}}+v_{t}\right)\left[\begin{array}{c}
2 u_{x} \\
u_{y}+v_{x} \\
u_{z}+w_{x} \\
0
\end{array}\right] \quad \mathbf{G}_{v}=-\left(\frac{1}{\operatorname{Re}}+v_{t}\right)\left[\begin{array}{c}
v_{x}+u_{y} \\
2 v_{y} \\
v_{z}+w_{y} \\
0
\end{array}\right] \quad \mathbf{H}_{v}=-\left(\frac{1}{\operatorname{Re}}+v_{t}\right)\left[\begin{array}{c}
w_{x}+u_{z} \\
w_{y}+v_{z} \\
2 w_{z} \\
0
\end{array}\right] .
\end{aligned}
$$



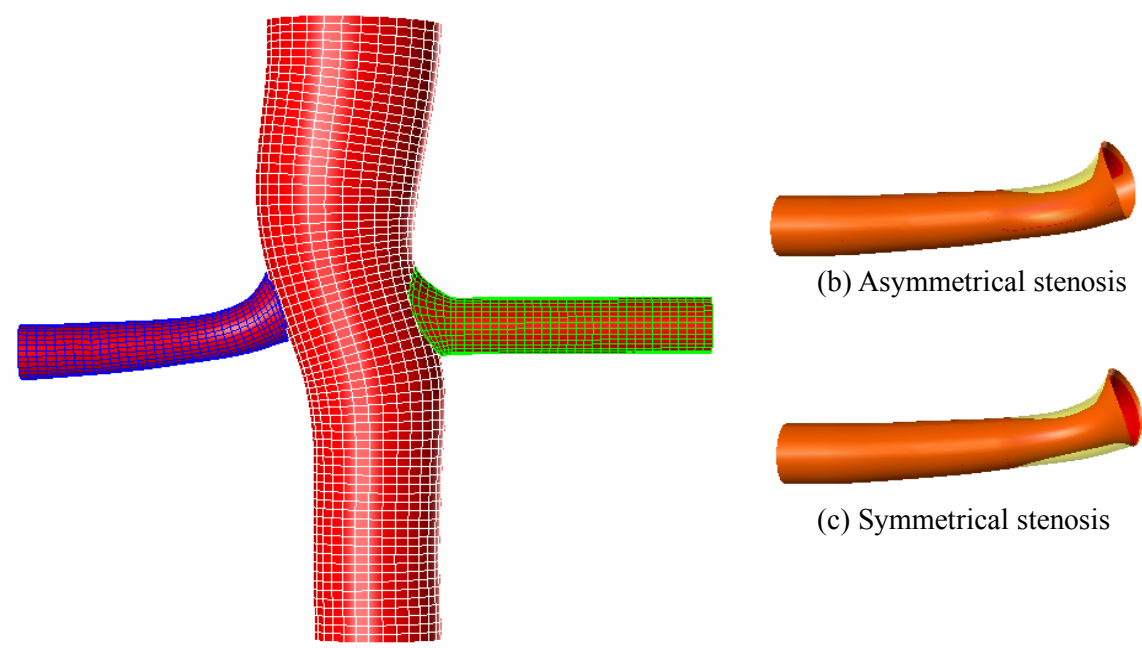

(b) Asymmetrical stenosis

(a) Mesh model

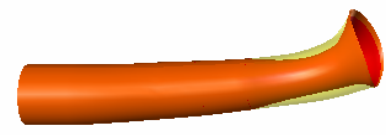

(c) Symmetrical stenosis

Fig.3 Computer model of the aortorenal bifurcation

Where $\beta$ is the peseudo-compressibility coefficient; $p$ is pressure; $u, v$, and $w$ are velocity components in the Cartesian coordinate system $x, y$ and $z$; Note that $\mathbf{q}$ associated with the pseudo-time is designed for an inner-iteration at each physical time step and will vanishes when convergence of computation is reached to satisfy the continuity equation. Re is the Reynolds number, and $v_{t}$ is the turbulent eddy viscosity. Note that because the flow to be considered was assumed to be laminar, the value of the turbulent eddy viscosity was set to be zero.

Time-dependent solutions of the incompressible Navier-Stokes equations were formulated with the finite volume method and were obtained in a time-marching manner using the pseudo-compressibility method. A third-order upwind differencing scheme, using the flux-splitting method, was implemented in a MUSCL fashion for the treatment of the convection terms. The viscous terms were evaluated by using a Gaussian-integration based second-order central differencing method. An implicit approximation-factorization method, based on the Euler implicit scheme, was employed for time integration. More details can be found in reference (10).

\subsubsection{Computer model of the aortorenal bifurcation}

The computer model of the aortorenal bifurcation was constructed based on the geometric parameters of the experimental model. In the present study, velocity profiles at the outlet boundaries are prescribed according to Poiseuille law for steady computation, and are defined by Womersley solution for unsteady computation, which requires an adequate length from the aortic inlet to each outlet to allow full development of the flow in each individual vessel. For this reason, the distal ends of the aortic trunk and renal branches were artificially extended downstream (Approximately 55 times the diameter of each outlet).

A structured grid scheme was employed to create the computational mesh model shown in figure 3(a) (the extensions are not shown). Herein, the inlet face of the vessel model was firstly meshed in an O-type grid pattern and then the face grid was mapped throughout the vessel domain along the center axis. Note that the aortic trunk and the renal branches were meshed separately considering that the present CFD software is a multi-block solver where fluid dynamic computations are performed separately for different blocks with the communications among them realized by the use of an overset-grid technique ${ }^{(6)}$.

Furthermore, two typical stenoses were artificially generated and implanted in the normal left renal artery by moving and transforming meshes in the relevant regions. Shown respectively in figures 3(b) and (c) are the computer models of two stenosed left renal arteries: (b) is a diseased left renal artery featured by an asymmetrical stenosis at the cranial 
wall near the branch entrance; the stenosis results in a maximum $25 \%$ reduction of the normal vessel cross-sectional area; herein it is named a mild RAS; and (c) is a left renal artery suffering from an axially symmetrical stenosis which leads to a maximum $50 \%$ decrease of the normal vessel cross-sectional area; such a stenosis is generally classified as a severe stenosis.

The dependence of computation on mesh density was studied by performing computations using $120 \times 31 \times 21(120, \quad 31$ and 21 denote the dividing amounts of computational mesh along the axis of vessel, and in the circumferential and radial directions of cross section, respectively), $170 \times 31 \times 21$, and $260 \times 31 \times 21$ grid systems, respectively. A comprehensive comparison among the computed results revealed that the $170 \times 31 \times 21$ grid system was capable of providing predictions of the key hemodynamic variables within $1 \%$ of those by using the $260 \times 31 \times 21$ grid system. With this understood, and at the same time allowing for the acceptable computational cost, the $170 \times 31 \times 21$ grid system was adopted for all the computations performed in the present study.

\subsubsection{Boundary conditions}

Boundary conditions for the aortorenal bifurcation were prescribed by flow waveforms (shown in figure 4) computed by a closed-loop lumped parameter model of the whole circulatory system $^{(11,12)}$. In order to enable the update of boundary conditions at intervals of an arbitrary time step during computation, the model-generated discrete flow waveforms were transformed into time-continual flow waveforms by means of Fourier series decomposition. The flow entering the abdominal aortic inlet is anatomically the flow leaving the outlet of the preceding aorta and hence can be approximated as a fully developed flow. Likewise, the outflows at the outlets can be described as fully developed flows considering that in the computer model the outlets are extended far away from the physical bifurcation region. The expression of Womersley solution was employed to define the pulsatile fully developed flow velocity profiles at the boundary faces.

\section{Results}

\subsection{Comparisons between experiment and computation}

Measurements of velocity profiles and wall shear stress were conducted under a flow condition characterized by a Reynolds number $(\mathrm{Re})$ of 800 at the aortic inlet with a flow division ratio (aorta inlet/branch outlet) of 0.25 for both the renal branches. The same flow condition was applied to the computation as well.

The velocity profiles measured at the common median plane are shown in figure 5(a), and the computed results are illustrated in figure 5(b). The computations agree reasonably well with the measurements for both the aortic trunk and the renal branches. The two renal branches show evident difference in the development of velocity profile along the vessels. This may be ascribed in part to the difference in the sites where the branches joint the trunk, and more prominently to the difference in the geometric structures of the two branches.

Figures 6(a) and (b) show the measured and the computed wall shear stresses along the 


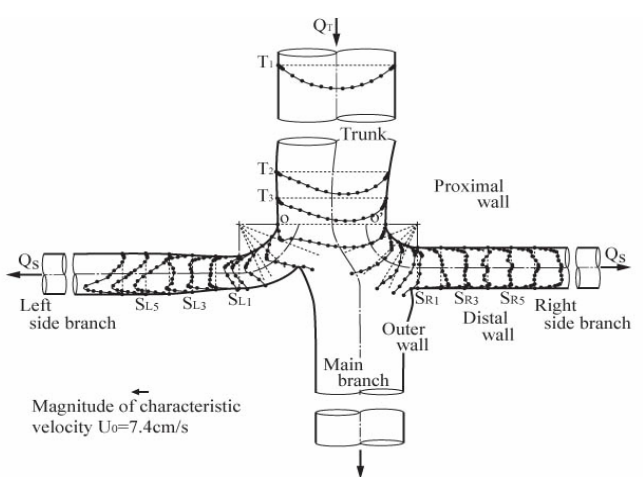

(a) Experiment

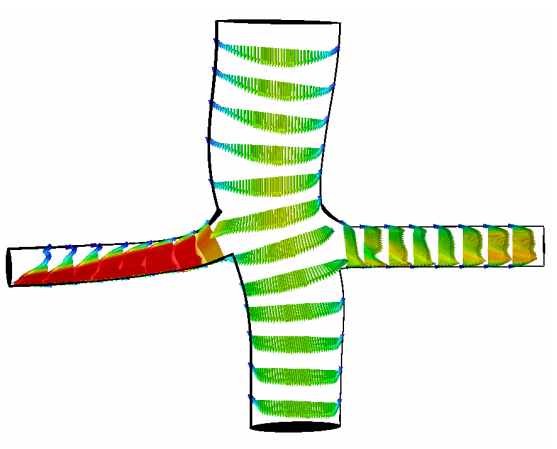

(b) Computation

Fig.5 Measured and computed velocity profiles

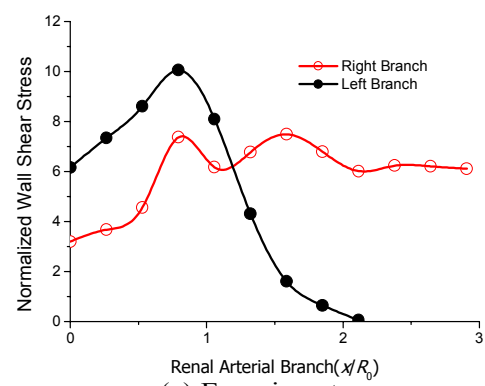

(a) Experiment

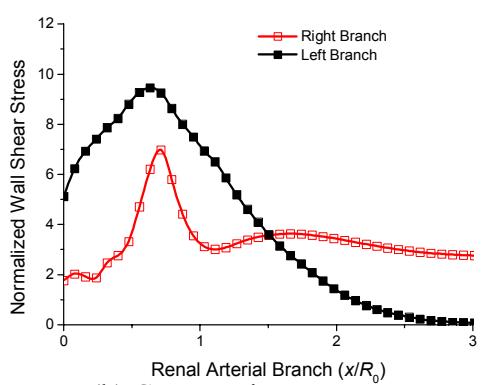

(b) Computation

Fig.6 Measured and computed wall shear stresses

proximal walls of the left and the right renal branches, respectively. Herein, the wall shear stresses are normalized by the shear stress at the aortic inlet wall. Though differing significantly in quantity, the experiment and the computation show similar wall shear stress distribution patterns along the two branches: the wall shear stresses decrease smoothly to approach a constant value after reaching a peak value along the right branch; whereas along the left branch the wall shear stresses decrease steeply towards zero after reaching a higher peak value. The considerable quantitative discrepancy between the experiment and the computation is very likely to stem from the failure to reproduce the details of the trunk-branch junctions during computer model construction, and is possibly attributable in part to the limit of accuracy in wall shear stress measurement.

\subsection{Computations under pulsatile flow conditions}

3.2.1 Hemodynamics at the normal aortorenal bifurcation

(1) Flow Velocity Patterns

Figure $7(\mathrm{a}, \mathrm{b})$ shows the computed velocity profiles in the median plane of the normal aortorenal bifurcation at two typical moments ((a) peak systole, (b) late systole), respectively. Based on these pictures and the computed results at other moments over a cardiac cycle, the basic characteristics of the transient fluid dynamics at the aortorenal bifurcation are outlined as follows. In early systole, relatively axisymmetrical cross-section velocity profiles are established and persist till peak systole in both of the renal branches. Thereafter, in late systole, rapid deceleration of flow elicits significant changes in the flow patterns. One of the most noticeable changes is the formation of flow separations near the proximal wall in both of the branches. The two branches, however, are not identical in the regions subject to flow separation. In the right branch, flow separations are present in a narrow region immediately after the branch entrance, whereas in the left branch, flow separations appear in a broad region downstream from the branch throat. Meanwhile, the axisymmetrical cross-section velocity profiles seen in early systole are gradually converted 


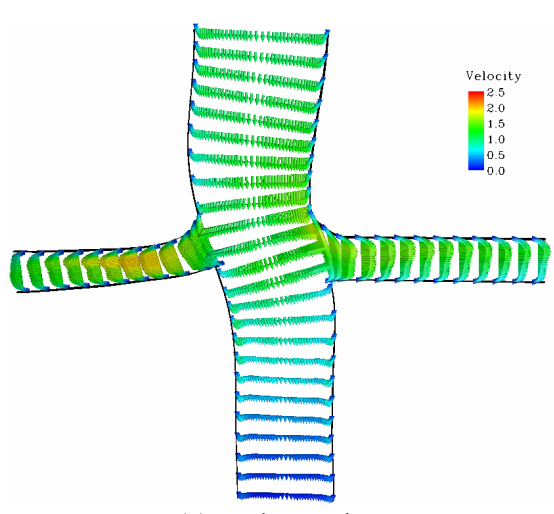

(a) Peak systole

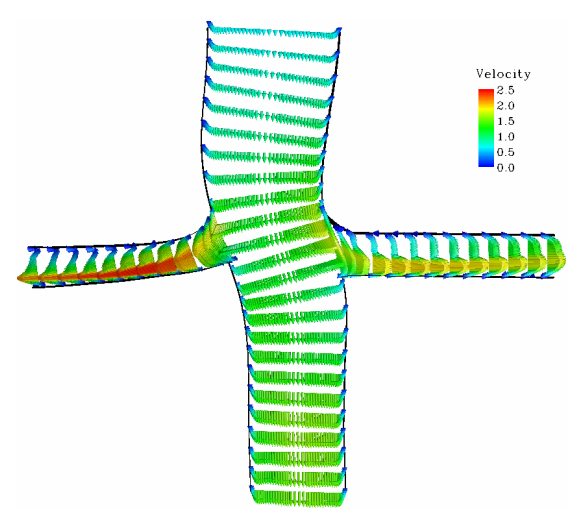

(b) Late systole

Fig.7 Computed velocity profiles in the median plane

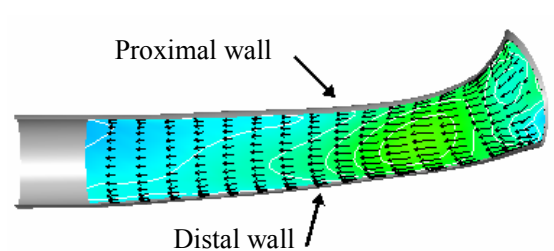

(a)Peak systole
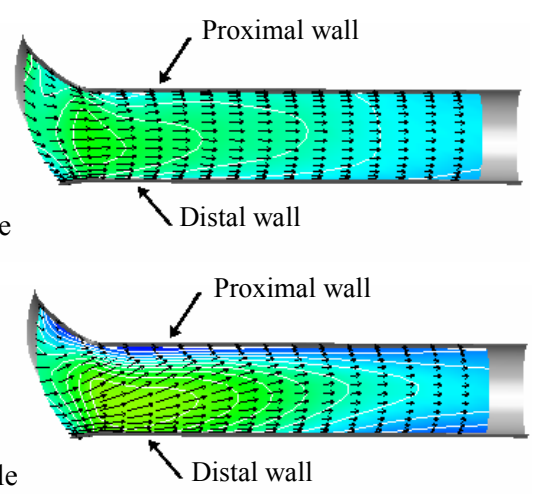

(b) Late systole

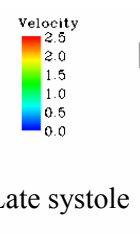

Fig.8 Computed velocity vector maps on the circumferential near-wall surfaces

into markedly asymmetrical ones characterized by high flow velocity near the distal walls and low flow velocity near the proximal walls. Subsequently in early diastole, symmetrical flow patterns are recovered and maintained till the end of the diastole in both of the branches.

Figures 8(a) and (b) illustrate the computed velocity vector distributions on the circumferential near-wall surfaces of the branches in peak systole and late systole, respectively. In peak systole, flows are fairly axisymmetrical; whereas in late systole, strong secondary flows indicated by flows directed from the distal wall side to the proximal wall side are observed in the upstream portions of the renal branches. An investigation over a cardiac cycle demonstrates that strong secondary flows take place exclusively in late systole.

\section{(2) Wall Shear Stress}

The computed color maps and contours of shear stresses in the renal branches are presented in figures 9(a, top view) and (b, bottom view) at three typical moments (from left to right: early systole, peak systole, and late systole), respectively. It is evident that the distal walls immediately after the branch entrance experience high wall shear stresses and large wall shear stress gradients (WSSG); whereas, the proximal walls nearby the flow separation region suffer from markedly low wall shear stresses, especially in late systole.

Considering the pulsatile feature of flow, a comprehensive understanding of the transient WSS entails the introduction of parameters that can synthesize the main characteristics of the transient WSS over a cardiac cycle. At this point, mean wall shear stress (MWSS) and oscillatory stress index (OSI) might be the most representative parameters fit for an integrative evaluation of the transient WSS. The former represents the average of the absolute value of the transient WSS over a cardiac cycle; and the latter reflects the oscillatory degree of WSS, for example, a purely unidirectional WSS is corresponding to an 


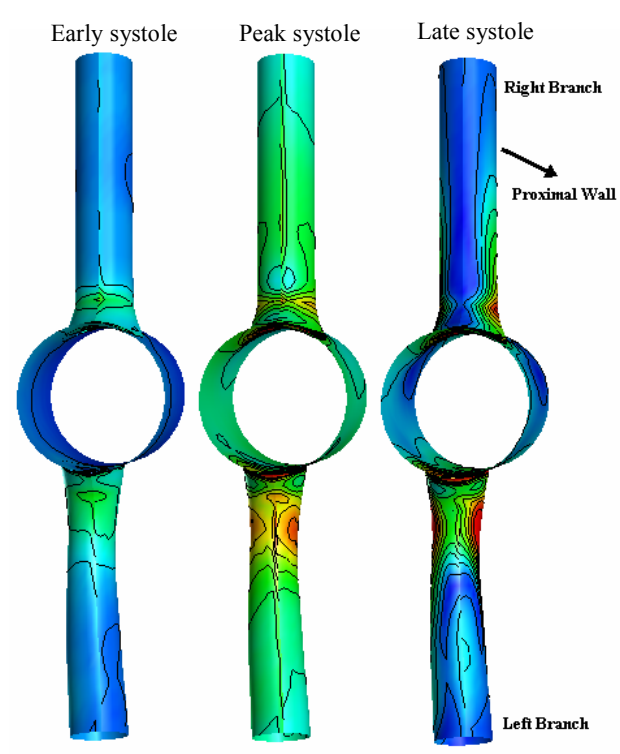

(a) Top view

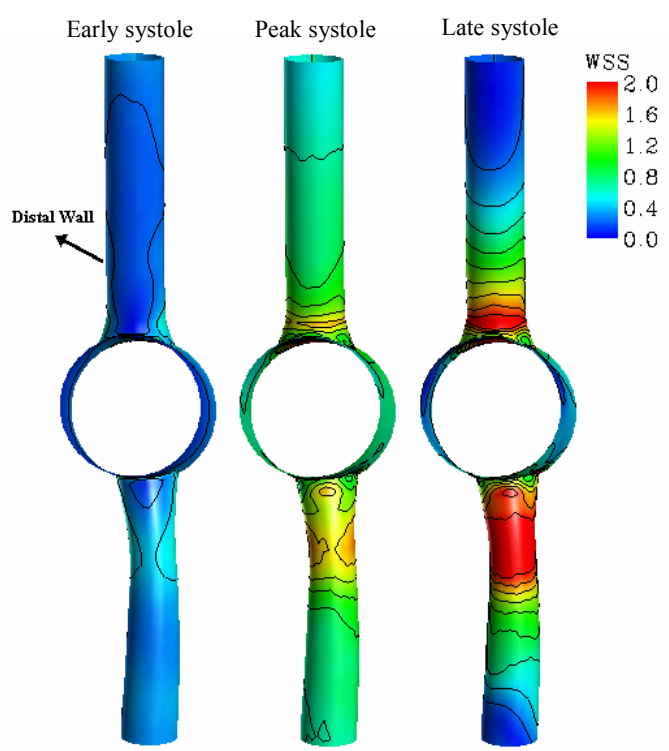

(b) Bottom view

Fig.9 Computed wall shear stress distributions

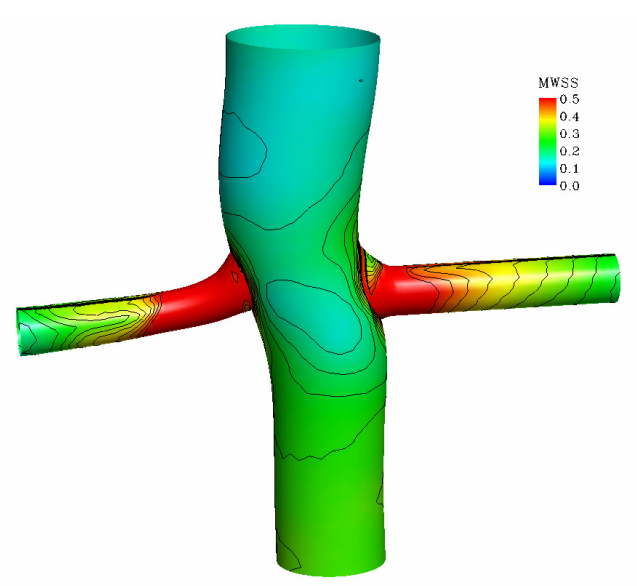

(a) MWSS

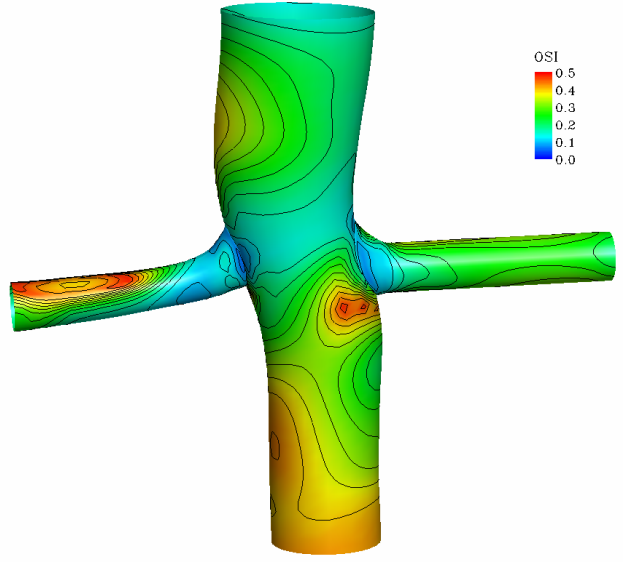

(b) OSI

Fig.10 Computed MWSS and OSI over a cardiac cycle

OSI value of 0 , in contrast, a strongly bidirectional WSS may result in a high OSI value up to 0.5. Figures 10(a) and (b) show the computed MWSS and OSI, respectively. A large part of the upstream portions of the two branches are present with high MWSS, except for a narrow region at the proximal wall immediately after the ostium of the right branch where low MWSS are observed. Another region exposed to markedly low MWSS is the proximal wall downstream from the throat of the left renal branch. For the aortic trunk, two low MWSS regions are predicted at the left and the right walls, respectively. With respect to OSI, evidently high OSI values are observed in the low MWSS region of the left branch, whereas no high OSI region is identified in the right branch. For the aortic trunk, high OSI regions correspond well with the low MWSS regions expect for the downstream portion where both high OSI and relatively high MWSS are predicted. If investigate the distribution patterns of MWSS and OSI in relation to the features of the intravascular flows, it is easy to find that the low-MWSS-and-high-OSI regions coincide well with the regions where flow separations are present.

3.2.2 Hemodynamics in the stenosed left renal branches

Computed velocity profiles in the median planes of two stenosed left renal arteries at two typical moments are illustrated in figure 11. In peak systole, the velocity profiles computed 
for the renal artery subjected to a mild asymmetrical RAS are not significantly different from those predicted for the normal renal artery; whereas, for the renal artery suffering from a severe symmetrical RAS, pronounced flow separations are predicted in the region downstream from the stenosis. In late systole, although both of the stenosed renal arteries are predicted with the presence of flow separations in the vicinity of the proximal wall downstream from the stenoses, the flow separations developed in the severely stenosed renal artery are stronger and influence a broader flow range in both axial and radial directions. In diastole, due to low flow rate, relatively symmetrical and stable flow patterns are formed and maintained in both the arteries.

Shown in figure 12 are the computed color maps and contours of MWSS and OSI for the stenosed renal arteries. The stenosed walls are found to be exposed to extraordinarily high MWSS and low OSI. In contrast, at the proximal walls downstream from the stenoses, markedly low MWSS accompanied by high OSI are predicted. Compared with the mildly stenosed artery, the artery with a severe stenosis undergoes higher MWSS at the stenosed wall and lower MWSS and OSI at the proximal wall downstream from the stenosis.
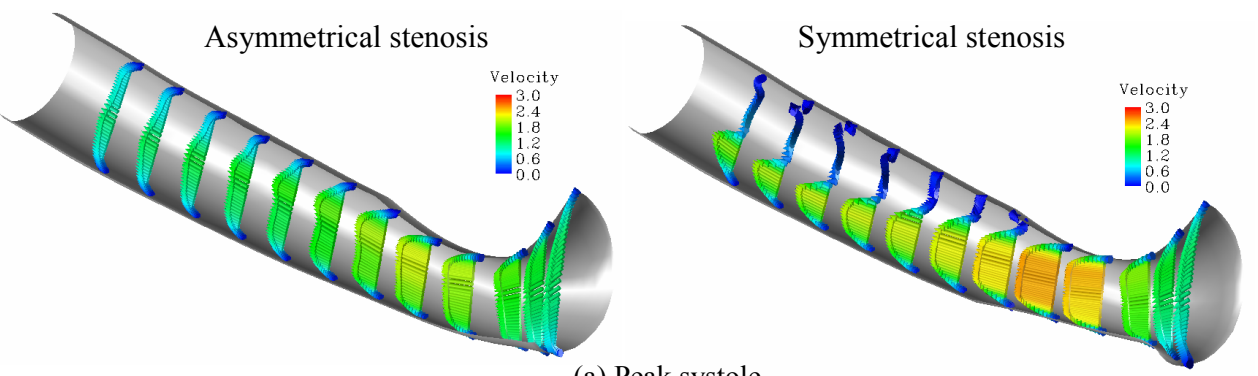

(a) Peak systole
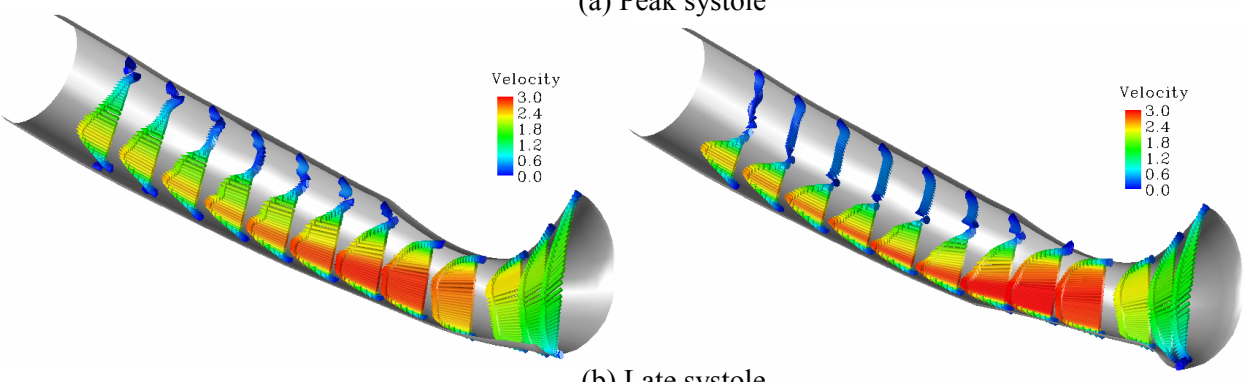

(b) Late systole

Fig.11 Computed velocity profiles in the median planes of the stenosed renal arteries
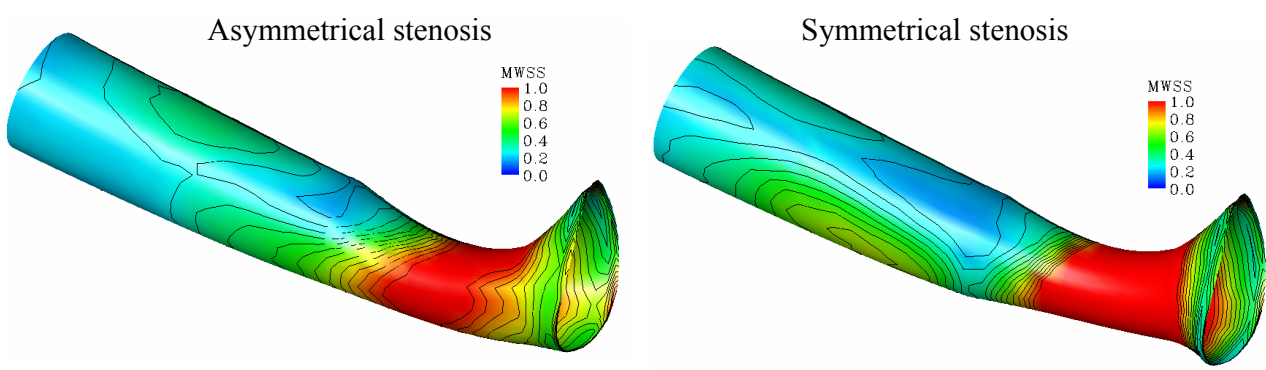

(a) MWSS

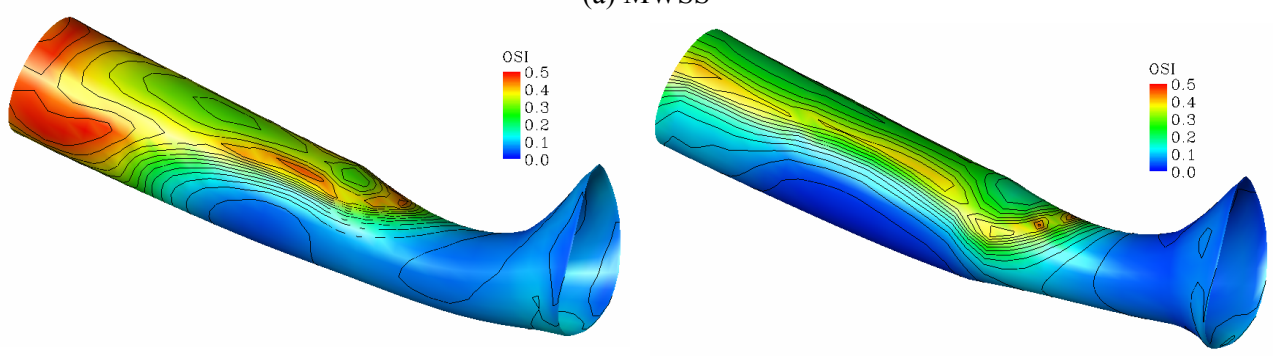

(b) OSI

Fig.12 Computed MWSS and OSI distributions on the stenosed renal arteries over a cardiac cycle 


\section{Discussion}

Although computations performed under pulsatile flow conditions provide a large pool of information on a variety of hemodynamic variables, in the following discussion, we will place primary attention on WSS, MWSS and OSI which were thought to play import roles in vascular disease. WSS exhibits a variety of patterns regarding the magnitude and the directionality; which raises a problem about what pattern of WSS is most likely to trigger vascular disease. This has been a longstanding topic being appreciated and argued on for decades. In 1985, Ku and his coworkers suggested that low oscillatory wall shear stress had the most intimate correlation with atherosclerotic plaque location ${ }^{(13)}$. Since the first proposal, this hypothesis has gained wide recognition and is now being supported by many up-to-date researches from various fields. In a recent review ${ }^{(14)}$, Snow systematically elucidated the association between WSS and the formation of atheroma and atherosclerosis in relation to Nitric oxide (NO), a ubiquitous and paracrine hormone thought to have an anti-atheroma action. He concluded that mean shear stress increased but pulsatile shear stress decreased NO production, thus the sites of greatest atherosclerosis development in the arterial system with low mean and high amplitude of shear stress were also the sites of low NO production. More recently, Himburg and the coworkers devised an experiment-simulation combined approach to assess the relation between the pulsatile WSS and the permeability of the endothelium to macromolecules ${ }^{(15)}$. Their study supported the hypothesis that regions of low shear stress or high OSI promoted the development of atherosclerosis via an increase in lipoprotein uptake. Simultaneously, they pointed out that although the combined action of MWSS and OSI might play a dominant role in determining endothelial permeability, it was necessary to bear in mind that the endothelial permeability is regulated by a complex interaction of spatially varying hemodynamic parameters including not only shear stress and OSI but also other parameters such as flow residence time and secondary flow. All these pioneering researches establish a substantial basis for this study to assess the correlation between local hemodynamic features and the localization of atherosclerosis in renal artery.

For the normal aortorenal bifurcation, computed results reveal that the proximal walls upstream of the right branch and downstream of the left branch are regions exposed to low MWSS and high OSI, the distal walls upstream of both the branches are regions undergoing large wall shear stress gradient (WSSG), and the lateral walls upstream of the two branches are affected by strong time-varying secondary flows. According to the hypothesis mentioned above, the proximal wall upstream of the right branch and the proximal wall downstream of the left branch dominated by low-MWSS-and-high-OSI combination are locations susceptible to atherosclerosis. Moreover, if taking into account the potential negative action of secondary flow and high WSSG on the endothelium function, the upstream portions of both the branches can be listed as candidates for locations apt to atherosclerosis as well. The marked difference between the two branches in the predicted locations prone to atherosclerosis indicates that geometric structure is an important determinant of the atherosclerosis-susceptible sites for a specific vessel.

For the stenosed renal arteries, computations reveal pronounced influence of RAS on the intravascular flow patterns, especially those downstream from RAS. Compared with the normal one; the renal artery suffering from a mild asymmetrical RAS exhibits lower MWSS and broader high OSI distributions at the proximal wall downstream of the renal artery. When the RAS develops into a severe symmetrical one, predictions reveal that at the proximal wall downstream of the renal artery, values of the MWSS are further lowered; and concurrently, the high OSI is significantly narrowed in distribution area and reduced in mean value. Then how will these RAS-induced hemodynamic changes affect the susceptibility of renal artery to atherosclerosis? And whether these changes act negatively or positively on the development of the existing RAS? In the light of the low oscillatory 
shear stress hypothesis, the asymmetrical-mild-RAS-induced lower MWSS and broader high OSI distributions may omen an increased risk of atherosclerosis in the region downstream from the stenosis. And in the case of the severe symmetrical RAS, the further lowered MWSS implies a further augment in the susceptibility of the corresponding region to atherosclerosis. Interestingly, it seems as if the narrowing of high OSI area and the reduction in mean value of OSI resulting from the severe symmetrical RAS may somewhat lessen the susceptibility of the low MWSS region to atherosclerosis. This, however, might not be the case because apart from MWSS and OSI some other hemodynamic variables may be involved in the initiation and progression of vascular disease. The most noticeable hemodynamic event induced by the severe symmetrical RAS is the pronounced flow disturbances featured by strong flow separations that persist for more than one half of a cardiac cycle and occupy a wide flow region downstream of the renal artery. Sustaining existence of flow separation implies a long flow residence time in the corresponding region. Himburg et al. pointed out the importance of residence time of solutes and formed elements of the blood in the neighborhood of vascular endothelium to the atherosclerosis process ${ }^{(15)}$. In this regard, the severe RAS might facilitate the development of atherosclerosis via the long flow residence time induced downstream from the stenosis. On the other hand, aside from the low oscillatory WSS known to affect atherosclerosis; unusually high WSS can activate platelets and thereby induce thrombosis ${ }^{(16)}$. In the present study, the severe RAS is predicted to double the MWSS at the stenosis throat.

\section{Conclusion}

The present study demonstrates that low oscillatory wall shear stress, the hemodynamic factor most closely associated with atherosclerosis, can be correlated well with flow separation; the distribution of low oscillatory wall shear stress exhibits strong dependence on vascular geometric structure; both the mild and the severe RAS lead to marked hemodynamic changes that are likely to promote the progression of the existing RAS.

However, this study is limited allowing for the rigid-wall assumption and the definition of identical boundary conditions for both the normal and the stenosed renal arteries. Deformation of arterial wall in radial direction is generally within $10 \%$ of arterial diameter, which therefore can be considered to exert a slight influence on intravascular fluid dynamics. In contrast, flow passing through a specific vessel may be significantly altered by variations in physiological and/or pathological conditions. For a renal artery with a progressing stenosis, flow waveform through it is not fixed but changes dynamically as a result of the renal autoregulation functioning to maintain the glomerular filtration rate.

Therefore, future work aiming at a physiologically realistic prediction of blood flow through renal artery, especially in the case when RAS is present, should carefully take the effect of the renal autoregulation into account. However, the renal autoregulation is a highly complex process involving multiple mechanisms; and its effect on hemodynamics is not limited to the renal circulation but covers the whole cardiovascular system ${ }^{(17)}$. Therefore, it seems very unrealistic to construct a high-dimensional computational system to represent the renal autoregulation with high accuracy. Eclectically, it is feasible to mimic the main features of the renal autoregulation with a system of mathematical functions. If further combine the functions with a hemodynamic model of the whole circulation, the global effect of the renal autoregulation can be accounted for. We have previously succeeded in simulating the hemodynamic responses to the Valsalva maneuver by constructing an integrative mathematical model of the cardiovascular system and the autonomic nervous regulatory system ${ }^{(12)}$. In this regard, establishing a similar mathematical model for the renal autoregulation seems possible and promising, the work, however, will be arduous considering that our current understanding of the renal autoregulation is yet limited and the clinical data able to support mathematical modeling is insufficient. 


\section{Acknowledgements}

This work is in part supported by a Grand-in-Aid for Scientific Research, No. (B)17300141, JSPS. Fuyou Liang is financially supported by the Rotary Yoneyama Memorial Foundation, Inc.

\section{References}

(1) Alejandro, R.C., et al., Distinct Renal Injury in Early Atherosclerosis and Renovascular Disease, Circulation, Vol. 106 (2002), pp.1165-1171.

(2) Rose, B.D., Pathophysiology of Renal Disease (1987), McGraw-Hill

(3) Yamamoto T., et al., Blood Velocity Profiles in the Human Renal Artery by Doppler Ultrasound and Their Relationship to Atherosclerosis. Arteriosclerosis, Thrombosis and Vascular Biology, Vol. 16(1996), pp.172-177

(4) Buchanana, J.R., et al., Hemodynamics Simulation and Identification of Susceptible Sites of Atherosclerotic Lesion Formation in a Model Abdominal Aorta. Journal of Biomechanics, Vol. 36(2003), pp.1185-1196

(5) Steinman, D.A., Image-based Computational Fluid Dynamics Modeling in Realistic Arterial Geometries. Annals of Biomedical Engineering, Vol. 30(2002), pp. 483-497

(6) Yamaguchi R., et al., Effect of Taper Angle on Wall Shear Stress at Tapered Right-angle Branch in Laminar Steady Flow. JSME International Journal (C),Vol.48(2005), pp.453-458

(7) Perktold, K., and Peter, R., Numerical 3D-simulation of Pulsatile Wall Shear Stress in an Arterial T-bifurcation Model, Journal of Biomedical Engineering, Vol. 12(1990), pp. 2-12

(8) Liu, H., et al. A Numerical Analysis of Renal Arterial Hemodynamics in a Medical Image-based Model, Transactions of JSME (A),Vol. 70(2004), pp.1247-1253

(9) Liang, F.Y., and Liu, H., A Multiscale Computational Study of Blood Flow in Human Renal Arteries, Proceedings of ASME 2005 SBC (2005), USA

(10) Liu, H., and Yamaguchi, T., Computer Modeling of Fluid Dynamics Related to a Myocardial Bridge in a Coronary Artery, Biorheology, Vol. 36(1999), pp.373-390

(11) Liang, F.Y., and Liu, H., A Closed-loop Lumped Parameter Computational Model for Human Cardiovascular System, JSME International Journal (C),Vol. 48(2005), pp.484-493

(12) Liang, F.Y., and Liu, H., Simulation of Hemodynamic Responses to the Valsalva maneuver: An Integrative Computational Model of the Cardiovascular System and the Autonomic Nervous System, Journal of Physiological Sciences, Vol. 56(2006), pp. 45-65

(13) Ku, D.N., et al., Pulsatile Flow and Atherosclerosis in the Human Carotid Bifurcation. Positive Correlation between Plaque Location and Low Oscillating Shear Stress, Arteriosclerosis, Vol. 5(1985), pp.293-302

(14) Snow, H.M., Atheroma and the Mechanics of Blood Flow in Arteries, Irish Journal of Medical Science, Vol. 171(2001), pp.165-169

(15) Himburg, H.A., et al., Spatial Comparison between Wall Shear Stress Measures and Porcine Arterial Endothelial Permeability, American Journal of Physiology, Vol.286 (2004), pp. 1916-1922

(16) Ku, D.N., Blood Flow in Arteries, Annual Review of Fluid Mechanics, Vol. 29(1997), pp. 399-434

(17) Holstein-Rathlou, N.H., and Marsh, D.J., Renal Blood Flow Regulation and Arterial Pressure Fluctuations: A Case Study in Nonlinear Dynamics, Physiological Reviews, Vol.74 (1994), pp. 637-680 